Article

\title{
Optimum Electrode Configurations for Two-Probe, Four-Probe and Multi-Probe Schemes in Electrical Resistance Tomography for Delamination Identification in Carbon Fiber Reinforced Composites
}

\author{
Luis Waldo Escalona-Galvis ${ }^{1}$ (D), Paulina Diaz-Montiel ${ }^{2}$ and Satchi Venkataraman ${ }^{1,2, * \text { (D) }}$ \\ 1 Computational Science Research Center, San Diego State University, San Diego, CA 92182, USA; \\ lescalonag21@gmail.com \\ 2 Department of Aerospace Engineering, San Diego State University, San Diego, CA 92182, USA; \\ pdiazmontiel@sdsu.edu \\ * Correspondence: satchi@mail.sdsu.edu; Tel.: +1-619-594-6660
}

Received: 28 February 2018; Accepted: 20 April 2018; Published: 24 April 2018

\begin{abstract}
Internal damage in Carbon Fiber Reinforced Polymer (CFRP) composites modifies the internal electrical conductivity of the composite material. Electrical Resistance Tomography (ERT) is a non-destructive evaluation (NDE) technique that determines the extent of damage based on electrical conductivity changes. Implementation of ERT for damage identification in CFRP composites requires the optimal selection of the sensing sites for accurate results. This selection depends on the measuring scheme used. The present work uses an effective independence (EI) measure for selecting the minimum set of measurements for ERT damage identification using three measuring schemes: two-probe, four-probe and multi-probe. The electrical potential field in two CFRP laminate layups with 14 electrodes is calculated using finite element analyses (FEA) for a set of specified delamination damage cases. The measuring schemes consider the cases of 14 electrodes distributed on both sides and seven electrodes on only one side of the laminate for each layup. The effectiveness of EI reduction is demonstrated by comparing the inverse identification results of delamination cases for the full and the reduced sets using the measuring schemes and electrode sets. This work shows that the EI measure optimally reduces electrode and electrode combinations in ERT based damage identification for different measuring schemes.
\end{abstract}

Keywords: electrical resistance tomography; carbon fiber reinforced composites; optimization; damage identification

\section{Introduction}

Carbon fiber reinforced polymer (CFRP) matrix composites are prone to intralaminar matrix cracking and interlaminar delamination failure due to the brittle nature of the matrix material. These damages change the effective electrical conductivity of CFRP composites. Imaging methods based on electrical resistance change such as Electrical Resistance Tomography (ERT) measure the internal conductivity change in a material indirectly from surface voltage measurements when electric currents are applied to the sample [1]. Damage modes in CFRP such as delamination and matrix cracks create measurable electrical resistance [2,3] or voltage changes [4]. Identification of specific damage modes must face several challenges since changes in volume fraction affect accuracy [5]. Electrical measurements significantly change as damage evolves towards different modes [6] and measurements must be localized for effective estimates of some modes [7]. Electric current magnitude and direction vary with respect to the electrode location $[5,8]$ and can become negligible at some 
interlaminar regions $[6,9]$, which, depending on the considered layup, will affect in-plane $[7,10]$ and normal-to-fiber damage location estimation [11].

Damage identification using ERT with high sensitivity and distinguishability requires a large number of sensors, which becomes difficult in practice for real applications due to hardware and computational effort. To maximize the measurement accuracy within the hardware limitations and computational time requirements in real-time sensing, it is necessary to optimally select sensing sites under appropriate measuring schemes for accurate damage identification using ERT.

The minimum number of electrodes necessary for damage identification using ERT is relative to the tested specimen $[2,10]$ and the best sensing combinations (surface or oblique resistance) depend on the extent of damage and loading conditions [6] that can represent a factorial increase in the number of combinations with the number of electrodes that must be optimized [11]. The geometric arrangement of the surface electrodes and the scheme used in the electrode combinations used for electric current injection and voltage measurement are crucial components of the sensing system design. When electric current is injected between two electrodes, voltage can be measured at this pair for direct resistance measurement using a two-probe [9] scheme, at electrodes located between the injection pair in a four-probe scheme $[8,12]$ or at all possible electrode pair locations in using a multi-probe scheme [4].

A number of papers have investigated electrode placement and sensing configurations for Electrical Resistance Tomography and Electrical Impedance Tomography (EIT), for example in biomedical [13], geophysical [14-16], and pipe flow [17-20] applications. These electrode placement designs are very problem and medium specific $[15,18-23]$. Some existing methods use geometric arrangements chosen intuitively based on knowledge of the problem and some prior experience $[13,19,24-26]$. In some cases, a priori chosen geometric arrangements were compared and evaluated using the reconstruction accuracy as a metric [22,25-27]. In these cases, the selection requires test data and is limited to choosing between a priori selected designs that satisfy hardware limitations.

In this work, we present a sensor optimization approach that differs from existing papers in two respects. First, it is tailored to composite laminates, which are unique in the fact that they are thin layered domains with discretely varying orthotropic properties from one layer to another. Second, the approach does not require problem insight or experience to choose a priori geometric arrangements. For any given problem, it simply needs to be able to identify exhaustively all possible sites one can have sensors or electrodes at, and from this can choose current electrode pairs and voltage measurement pairs using a numerical model of the problem using an "Effective Independence" (EI) measure [11] described later in this paper. We perform optimal electrode combination selection using the EI measure for ERT based damage identification of delamination cracks on laminates with different stacking sequences considering two-probe, four-probe and multi-probe measuring schemes.

\section{Problem Formulation and Solution}

The governing differential equation for the voltage $(u)$ in a body with conductivity distribution $\sigma$ under the spatial coordinates $\bar{r}$ is given by the following steady state diffusion equation and associated boundary conditions $[1,28]$ :

$$
\begin{gathered}
-\nabla \cdot(\sigma \nabla u)=0, \quad \bar{r} \in \Omega, \\
\int_{e l} \sigma \frac{\partial u}{\partial \bar{n}} d S=I_{l}, \quad \bar{r} \in \partial \Omega_{e l}, \\
\sigma \frac{\partial u}{\partial \bar{n}}=0, \quad \bar{r} \notin \partial \Omega_{e l} .
\end{gathered}
$$

The first boundary condition expressed in Equation (2) relates the current density over the $l_{t h}$ electrode (surface) to the electric current $I_{l}$ passed through the electrode ( $\bar{n}$ is the outward unit normal) defined by the surface integral over the electrode $(e l)$ on the boundary $\partial \Omega_{e l}$. The second boundary condition expressed in Equation (3) specifies the electrode-free insulated boundaries (regions not included in the boundary $\partial \Omega_{e l}$ ). In orthotropic CFRP composites, the conductivity $\sigma$ varies in each 
layer due to ply orientation, and delamination within the laminate introduces free surfaces within the domain.

\subsection{Finite Element Model}

The governing equations for the electric potential distribution in a composite laminate with orthotropic plies were solved using ANSYS [29] FEA softwar (Academic Research, Release 15.0.7, Canonsburg, PA, USA). A simplified 2D planar model for the laminate cross-section that spans the thickness and length of the laminate is analyzed under a specified current injection to obtain the voltage distribution (Figure 1). The specimen, electrodes and the embedded delamination are all assumed to span the entire depth in the out of plane direction.

A layerwise model is used to analyze 16-ply laminates with and without delamination crack damage. Delamination cracks were created as free surfaces using doubly-defined nodes at the crack interface. The domain mesh consisted of eight node quadrilateral planar electric elements (PLANE 230). A mesh convergence study was performed and the element mesh size was found to be $0.125 \mathrm{~mm}$, which completely spans through the thickness of each ply. This resulted in a model with 27,044 elements.

Orthotropic electrical properties were used [11] to model $\left[0_{4} / 90_{4}\right]_{s}$ and $\left[(0 / 90)_{4}\right]_{s}$ cross-ply laminates. The conductivity values used for CFRP correspond to a graphite epoxy composite lamina with $62 \%$ fiber volume fraction [9]. The electrode material (silver) was modeled as isotropic with a $62.9 \times 10^{6} \mathrm{~S} / \mathrm{m}$ conductivity.

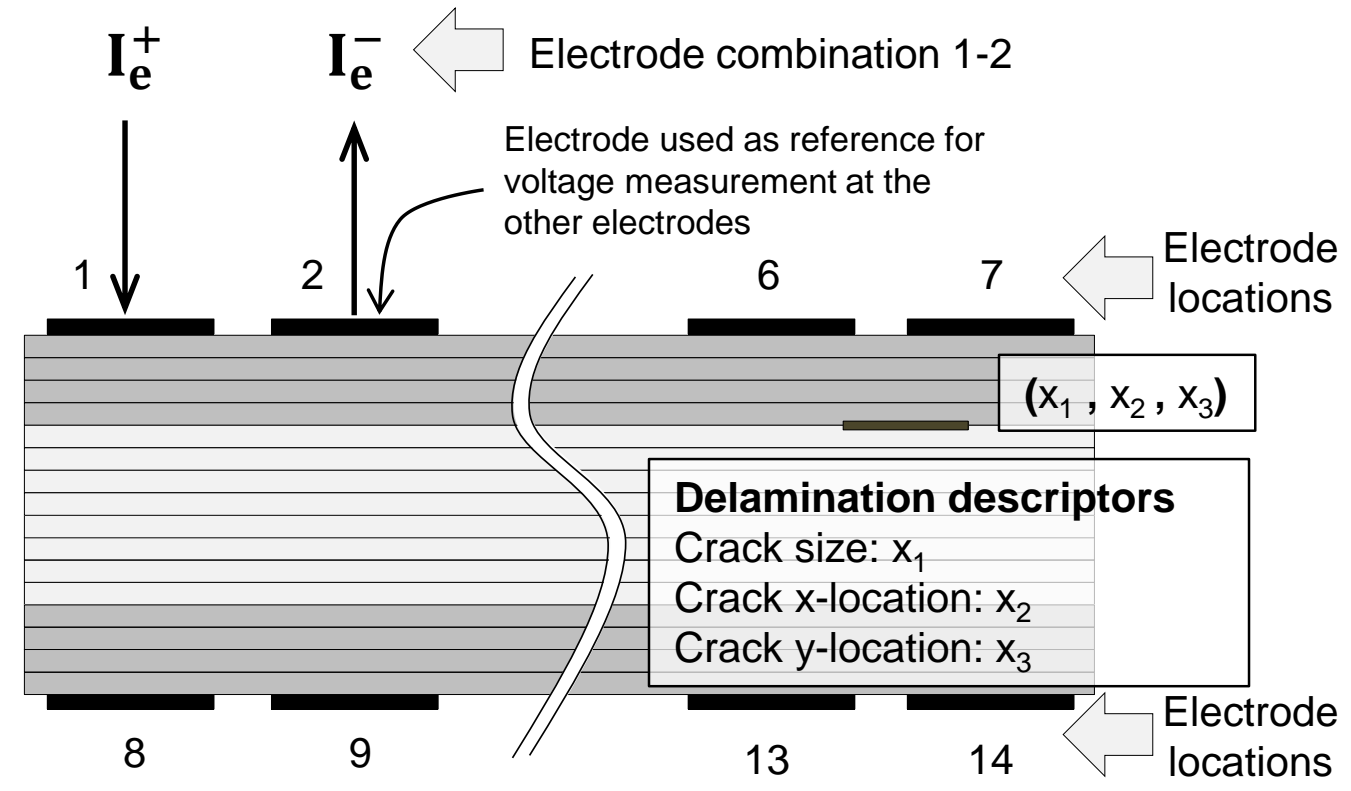

Figure 1. Schematic representation of a $\left[0_{4} / 90_{4}\right]_{S}$ composite laminate under damage with 14 electrodes where electric current is injected in pair 1-2.

\subsection{Measuring Schemes}

In ERT based detection, in general, there are multiple surface electrodes. The schemes for measurements refer to location of the electrode pairs for passing electric current and the locations of the electrode pairs for voltage sensing, relative to the chosen current electrodes. In this section, we discuss the geometric arrangement for the electrode locations corresponding to various schemes such as two, four and multi-probe schemes, and describe the geometric arrangement for the electrode locations.

In a general electrode setting, a specified current is injected between a chosen pair of electrodes and voltages at electrodes can be measured. In this study, the method will be demonstrated using a 2D plane representation of a laminate with $h=14$ electrodes distributed on the top and bottom surfaces of the laminate. This results in a total of $p=C_{2}^{h}=91$ possible electrode pairs for passing current. 
When the electric potential $(V)$ is measured between the same electrode pairs through which the electric current $(I)$ is applied, then this is referred to as a two-probe (Figure 2). The effective resistance is then computed using Ohm's Law as the ratio $V / I$ [9]. For a given damage case, the voltages measured at all possible electrode combinations are grouped as a vector, hereafter referred to as responses $\left(v_{s}\right)$. The matrix A shown in Figure 2 is the collection of responses corresponding to $N_{D}$ different damage cases.

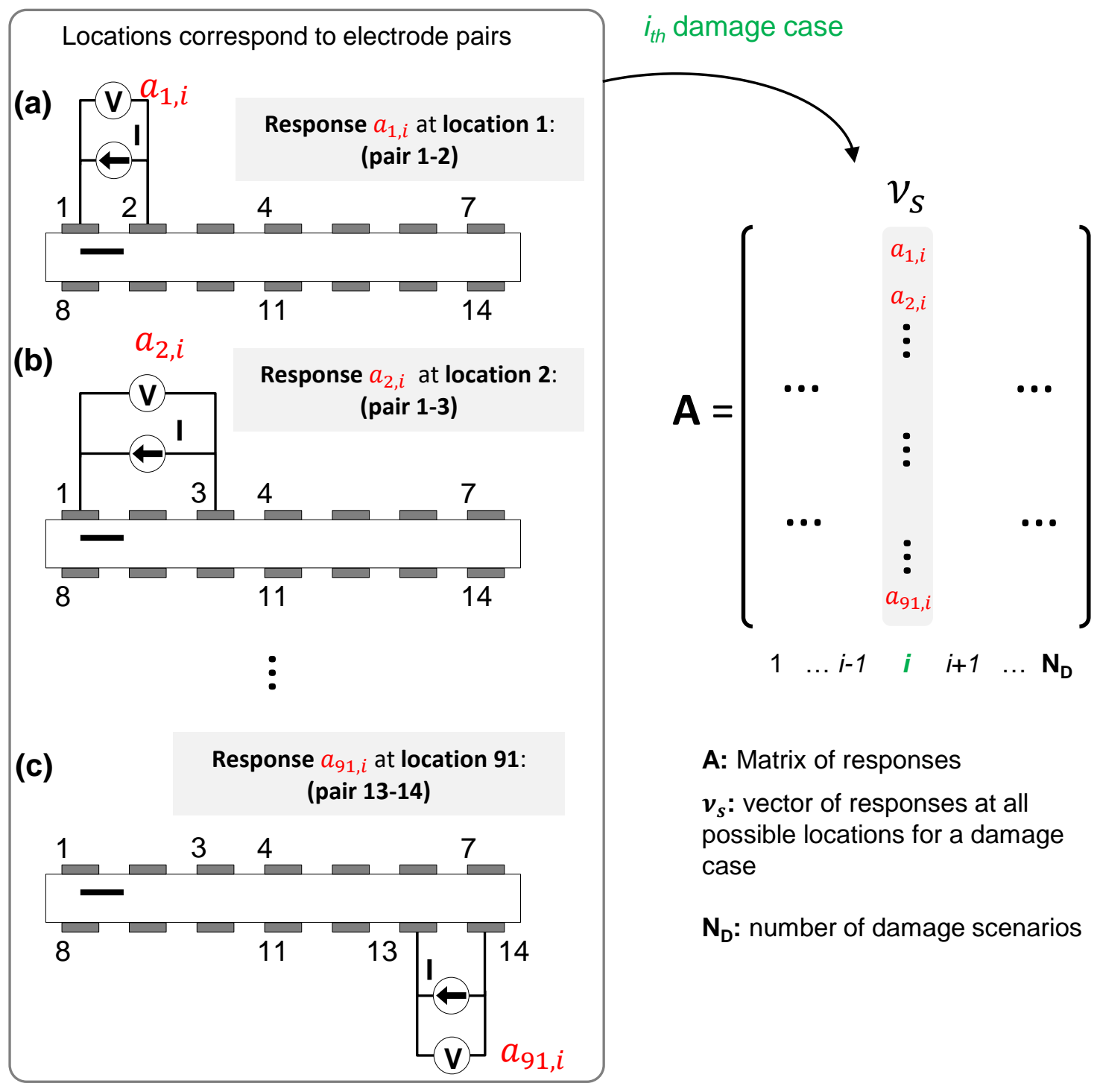

Figure 2. Scheme used for damage identification using two-probe schemes and two-sided electrodes. The matrix of observations $A$ is obtained from the responses in order to apply effective independence. Responses are grouped by damage scenario in column vectors $\left(v_{s}\right)$ in matrix $A$ : (a) starting with the response for consecutive electrodes on the left edge at the top surface; (b) following with increase of electrode distance and (c) finishing with the electrode pair on the right edge at the bottom surface.

If the electric potential $V$ is measured at a pair of electrodes that are physically located between the electrode pairs used for current injection (Figure 3), then this is referred to as a four-probe [12]. The ratio of the voltage measured at the electrode pair to the current injected can provide resistance $R=V / I$ [8]. In the four probe scheme, the electrodes for voltage measurements are always located within the physical horizontal space between the two current electrodes. When the two current electrodes are on 
the same side as in Figure 3a, then the voltage electrodes are also kept on the same side. When electric current and voltage are measured on the same face, we refer to this as surface resistance (Figure 3a,b). For example, when the current electrode pair is $1-5$ (as in Figure $3 b$ ), the possible choices for voltage electrode pairs are 2-3, 3-4, and 2-4. The larger spacing between electrodes for voltage measurements can sense changes farther away from the surface. When two current electrodes are selected to be on opposite faces, then the voltage measurements' electrodes are also selected on the opposite faces, but within the range of the current electrodes. For example, if the current electrodes are chosen to be 4 and 8 , then possible choices for voltage measurements are 2-10 and 3-9. When current injection and voltage measurement follow a diagonal path, we refer to this as equivalent oblique resistances (Figure 3c).

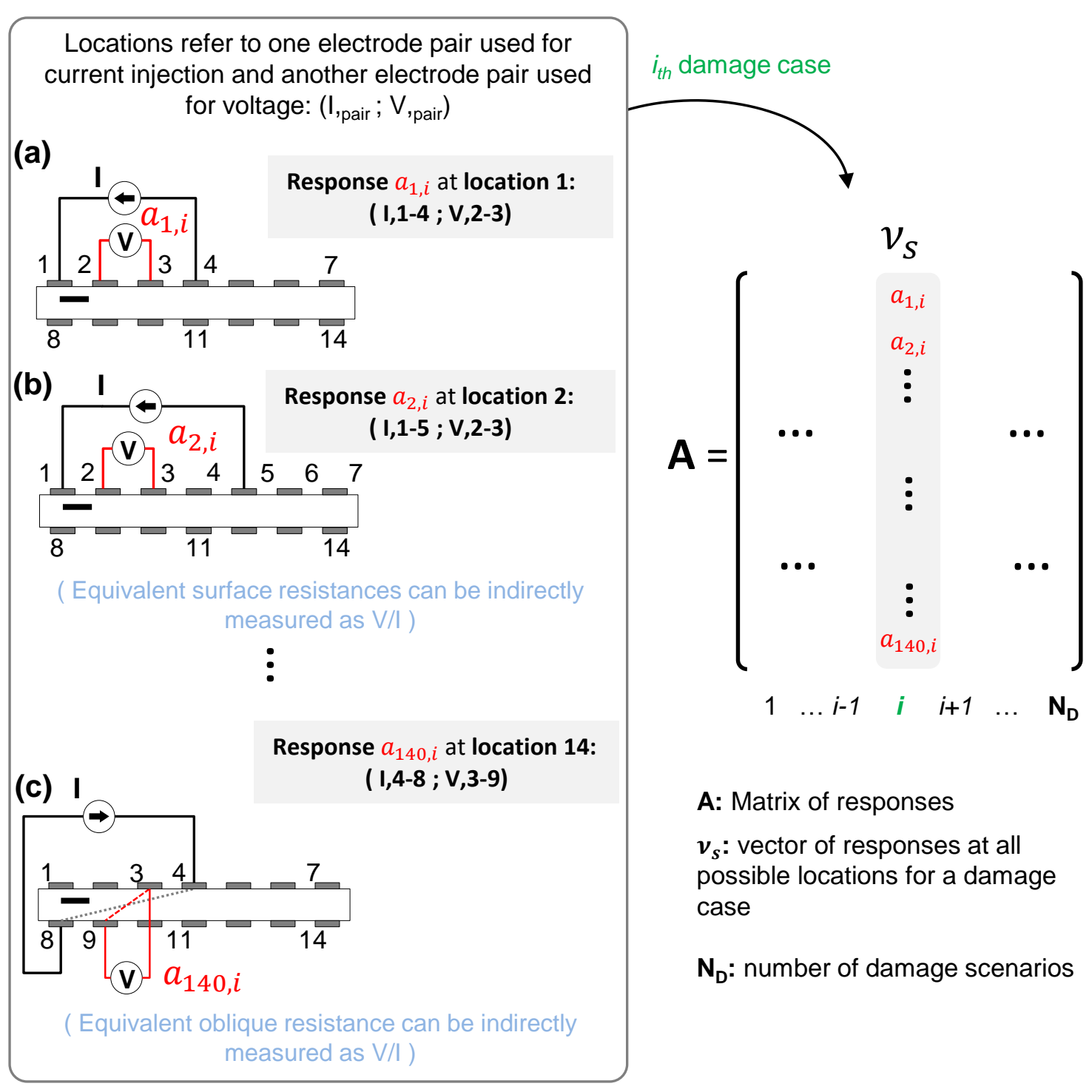

Figure 3. Scheme used for damage identification using four-probe schemes and two-sided electrodes. The matrix of observations $A$ is obtained from the responses in order to apply effective independence. Responses are grouped by damage scenario in column vectors $\left(v_{s}\right)$ in matrix $A$ : (a) starting on the top surface with schemes for surface resistance; (b) following with an increase of electrode distance between electric current electrodes and (c) finishing with schemes used for oblique resistance. 
For each of the $p=C_{2}^{h}$ current electrode injection pairs from the total set of electrode pairs, we can measure electric potentials at $(h-1)$ sites with respect to one of the current electrodes used as a reference electrode (Figure 4). This scheme is referred to as the multi-probe method [4].

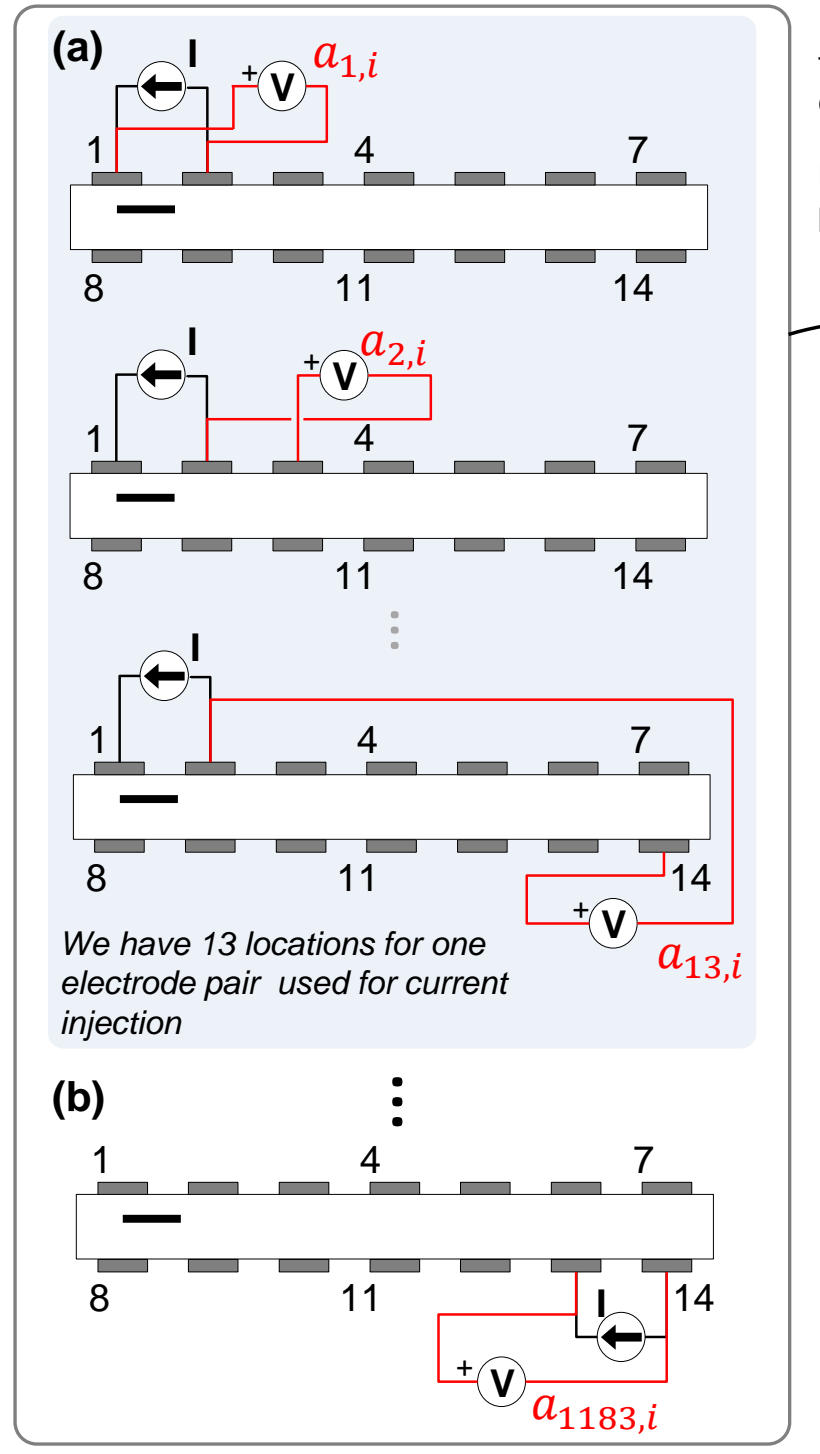

Electric potentials are obtained with respect to a reference electrode set at the pair for current injection.

Locations are referred to current injection pair and an electrode chosen for voltage

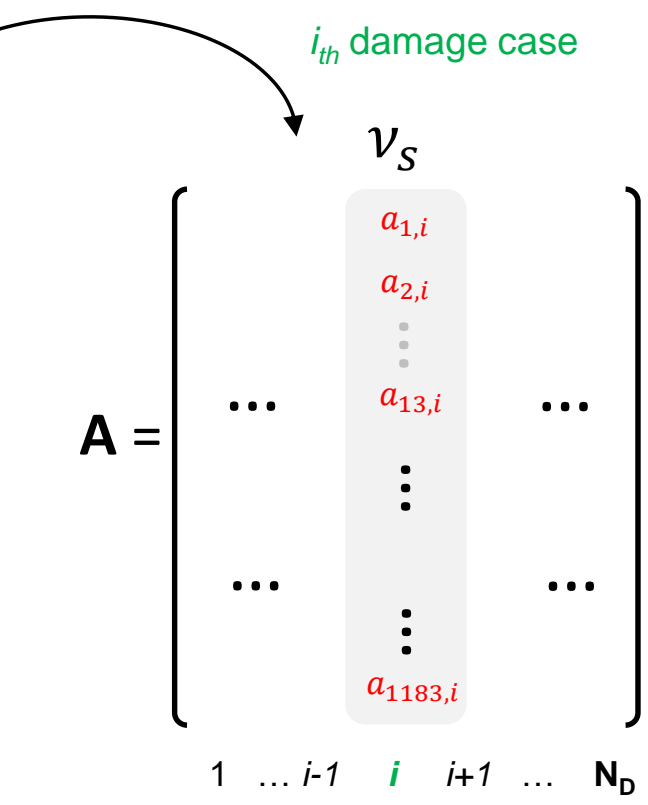

A: Matrix of responses

$v_{s}$ : vector of responses at all possible locations for a damage case

$\mathbf{N}_{\mathrm{D}}$ : number of damage scenarios

Figure 4. Scheme used for damage identification using multi-probe schemes and two-sided electrodes. The matrix of observations $A$ is obtained from the responses in order to apply effective independence. Responses are grouped by damage scenario in column vectors $\left(v_{s}\right)$ in matrix $A$ : (a) for an electrode pair used in current injection, voltages in all electrodes but the reference (e.g., electrode 2) will be associated with different responses; (b) the last response is the thirteenth response associated with the electric current injection pair on the rightmost bottom surface.

The total number of possible measurements using the measuring schemes on a composite laminate with a total of $h$ electrodes distributed either on one side or both sides are summarized in Table 1.

The goal of this paper is to present a method for optimal selection of electrode pairs for current injection and voltage measurements to perform ERT. 
Table 1. Number of measurements/combinations for the measuring schemes used for ERT.

\begin{tabular}{cccc}
\hline Scheme & $\begin{array}{c}\text { Number of } \\
\text { Measurements (Ns) }\end{array}$ & $\begin{array}{c}\text { Total for One-Sided } \\
\text { Electrodes }(\boldsymbol{h}=\mathbf{7})\end{array}$ & $\begin{array}{c}\text { Total for Two-Sided } \\
\text { Electrodes }(\boldsymbol{h}=\mathbf{1 4})\end{array}$ \\
\hline Two-probe & $C_{2}^{h}$ & 21 & 91 \\
Four-probe & $k \times \sum_{i=1}^{h-3}\left[\sum_{j=2}^{h-(i+1)} C_{2}^{j}\right]$ & $35 ; k=1$ & $140 ; k=4$ \\
Multi-probe & $(h-1) \times C_{2}^{h}$ & 126 & 1183 \\
\hline
\end{tabular}

\subsection{Effective Independence Based Electrode Selection}

This section presents a brief overview of the EI measure and its implementation for electrode selection. The use of EI measures has been previously demonstrated for optimum placement of sensors (accelerometers) for measuring structural vibration modes [30,31].

Let $v$ be the measured responses at all possible locations. A modal representation of $v$ is given in terms of matrix $\Phi$ of modal vectors and the vector $\chi$ of target modes by:

$$
v=\Phi \chi
$$

When performing tests, we have measurements of the response at finite sample points from which the modal coefficients have to be estimated. The measurements usually also have some noise, denoted here as $N$. The responses at measurement sites $v_{s}$ can be expressed using the modal representation, with modal vectors restricted to sensing sites $\Phi_{s}$, as shown in Expression (5):

$$
v_{s}=\Phi_{s} \chi+N .
$$

If we can assume the responses measured at sampling sites $v_{s}$ have uniform and uncorrelated Gaussian noise $N$ with with variance $\sigma^{2}$, the covariance matrix of estimation errors for the target mode response $\chi$ from the measured responses $v_{s}$, is obtained by the expected value (E) in Expression (6) [30]:

$$
P=E\left[(\chi-\hat{\chi})(\chi-\hat{\chi})^{T}\right]=\left[\Phi_{s}^{T}\left(\sigma^{2}\right)^{-1} \Phi_{s}\right]^{-1}=Q^{-1} .
$$

Minimizing the above covariance matrix is equivalent to maximizing the determinant of the Fisher Information Matrix (FIM) $(Q)$ leading to the best estimate $\hat{\chi}$. In the case where measurement noise is uncorrelated with identical statistical properties, the FIM can be expressed analogously as $A_{0}[30]$ in Expression (7):

$$
Q=\left(\sigma^{2}\right)^{-1} \Phi_{s}^{T} \Phi_{s}=\left(\sigma^{2}\right)^{-1} A_{0} \Rightarrow A_{0}=\sum_{i=1}^{d} \phi_{s}^{i T} \phi_{s}^{i}
$$

In the expression above, $\phi_{s}^{i T}$ corresponds to the $i_{t h}$ row in matrix $\Phi_{s}$. Deleting a row in $\Phi_{s}$ is equivalent to eliminating a sensor location and it removes information from the equivalent FIM [31] $\left(\Phi_{s}^{T} \Phi_{s}\right)$. The determinant of the new FIM $\left(Q^{i}\right)$ after deleting the $i_{t h}$ row can be expressed in terms of the original FIM [31]:

$$
\operatorname{det}\left(Q^{i}\right)=\operatorname{det}(Q) \times\left(1-E_{i}\right),
$$

where $E_{i}$ is the contribution of the removed sensor to the independent information of $\Phi_{S}$. The contribution of each sensor derived from the eigenvalues and eigenvectors of matrix $Q$ [11]. The ranked vector of eigenvalues $E_{D}$ provides the Effective Independence values. This effective independence distribution vector $E_{D}$ is the main diagonal of the effective independent (EI) matrix $E$ in Expression (9):

$$
E_{D}=\operatorname{diag}\left(\Phi_{s}\left[\Phi_{s}^{T} \Phi_{s}\right]^{-1} \Phi_{s}^{T}\right) .
$$


The computed EI values allow us to rank the sensor locations. After the sensor location with the lowest EI value is eliminated, the $E_{D}$ vector is recomputed and then iteratively used to eliminate the sensor location with the lowest contribution.

In this paper, we use the EI based method to investigate optimal selection of electrode pairs for the three schemes discussed. The selection requires a numerical model of the forward solution for the electrical conduction problem for the domain of interest. For our demonstration problems, voltage responses were obtained numerically from finite element analyses. The first step of the process is to construct a modal representation of the voltage responses. This is performed numerically. A set of preselected 810 delamination cases obtained from a full factorial (FF) Design of Experiments (DOE) by varying the three variables, namely crack length, crack center location along the $x$-axis and crack location along the $y$-axis. Since delamination cracks in composites are at interply locations, this variable is discrete. A matrix of responses $A_{N s \times 810}$ is obtained from the numerical simulations. These numerical simulations are then used to obtain the modal matrix $\Phi_{s}$ that provides the orthonormal basis for the response $v_{s}$ using a Singular Value Decomposition and dimension reduction on $A_{N s \times 810}$ [11]. Once the modal matrix or orthogonal basis $\Phi_{s}$ is available, one can proceed to calculation of the Effective Independence (EI) measures.

With the EI measures obtained from $\Phi_{s}$, measurement reduction consists of eliminating the row from $\Phi_{s}$ with the lowest EI value. The reduction process is performed iteratively by computing the $E_{D}$ values and updating the matrix $\Phi_{s}$ by eliminating the row associated with the sensing location with the lowest EI. The termination criteria can be set as given a number of sensors or measurements retained or when the determinant of $\left(\Phi_{s}^{T} \Phi_{s}\right)$ is less than a threshold value $\epsilon$ (between $10^{-9}$ and $10^{-2}$ ).

In essence, the EI based selection scheme starts with the complete set of all possibilities and eliminates sites with low contribution until the desired set is obtained.

Since the process of iteratively computing the equivalent FIM for EI based selection implies computation of matrix inversion of the FIM, the condition number of this matrix must be monitored during the process of row elimination from matrix $\Phi_{s}$. The initial condition number of the FIM was 1 for all studied cases. After applying the EI based reduction on the different schemes and layups, the condition number increased to a maximum of 13 with a typical final value below 10 . This results show that small numerical error is expected from the EI based reduction process.

\subsection{Electrode Combinations Reduction}

In this section, we will discuss the results of the EI based selection of electrodes of the three measurement schemes (two-, four- and multi-probe) discussed previously. For each case, the optimal electrodes for one-sided and two sided placements of electrodes are discussed. The technique is demonstrated for two crossply laminate designs, one with blocked plies $\left[0_{4} / 90_{4}\right]_{s}$ and the other with distributed plies $\left[(0 / 90)_{4}\right]_{s}$. Figure 5 presents the results for the two-probe scheme. The results for electrode selection are summarized by a heatmap plot. The heatmap plot is a $14 \times 14$ colored grid that represents the values of EI measures for the electrode combinations before and after EI based electrode reduction. The lower triangle of the square grid shows the EI measures for the current electrode pairs (rows)-voltage electrode pairs (columns) for the full set of electrodes. The diagonal and upper triangle of the grid represents the EI of the optimal set of electrodes after EI based reduction. The EI values of the omitted electrode pairs are set to zero.

Figure $5 \mathrm{a}, \mathrm{c}$ show the results of the retained electrodes under the two-probe scheme for the $\left[(0 / 90)_{4}\right]_{s}$ laminate for one-sided and two-sided electrodes, respectively. For the two-probe method, using an EI based selection on this stacking sequence resulted in a reduced set with 12 out of 28 electrode pairs for the setting with one-sided electrodes and a reduced set with 34 out of 91 pairs for the setting with two-sided electrodes.

Figures $5 \mathrm{~b}$,d show the results of the retained electrodes under the two-probe scheme for the $\left[0_{4} / 90_{4}\right]_{s}$ laminate for one-sided and two-sided electrodes, respectively. When sensor selection is applied on this stacking sequence, a reduced set with 13 out of 28 electrode pairs was obtained for the 
setting with one-sided electrodes and a reduced set with 32 out of 91 electrode pairs for the setting with two-sided electrodes.

(a)
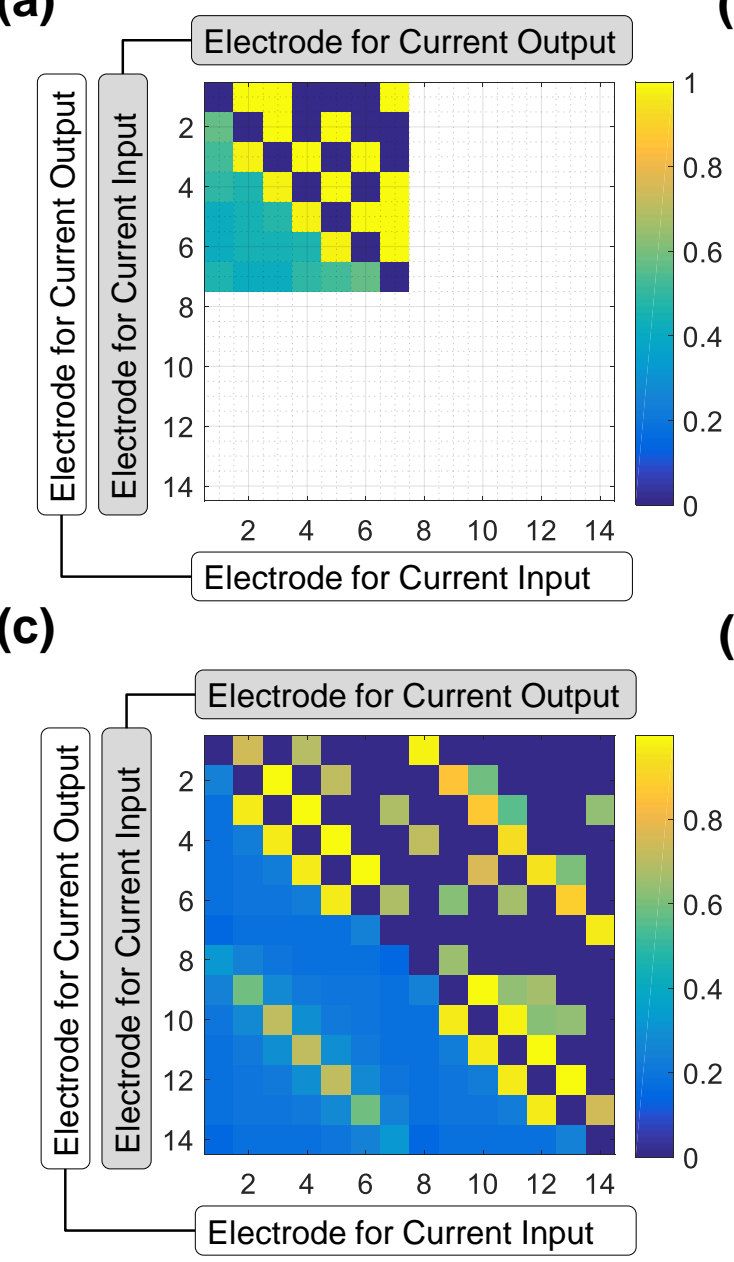

(e)

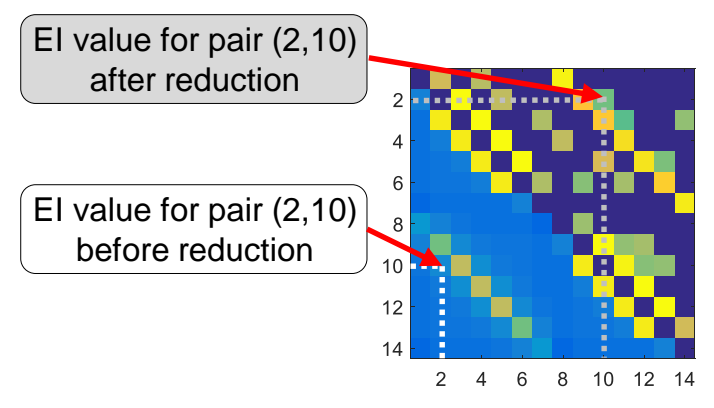

(b)

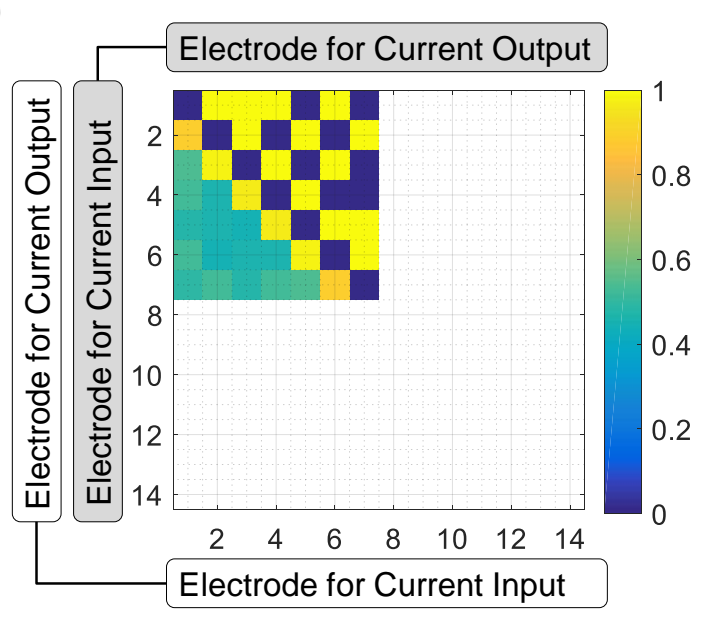

(d)
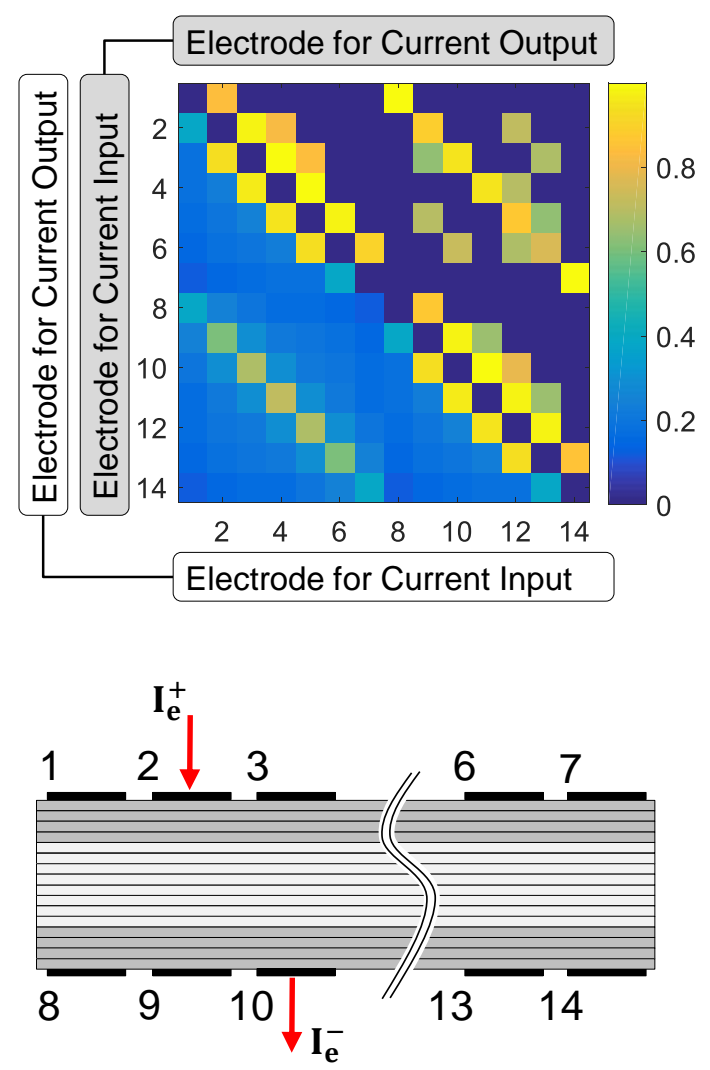

Figure 5. Results of Effective Independence (EI) for two-probe schemes using (a) one-sided electrodes on $\left([0 / 90]_{4}\right)_{s}$ layup; (b) one-sided electrodes on $\left[0_{4} / 90_{4}\right]_{s}$ layup and (c) two-sided electrodes on $\left([0 / 90]_{4}\right)_{s}$ layup and (d) two-sided electrodes on $\left[0_{4} / 90_{4}\right]_{s}$ layup. An example of the EI independence before and after reduction is shown in (e)

For both layups using the two-probe scheme, the full sets of combinations show that consecutive electrodes (e.g., 1-2, 2-3, 3-4...) have the highest contributions for both one and two face electrode placements. These combinations are preserved in the reduced sets. In the case of 14 electrodes, electrode pairs with vertically opposed placements also show high contributions before and after reduction. 
Figures 6 and 7 show the EI values for the reduced and optimum set of responses for all possible electrode pairs (abcissa) and the corresponding feasible voltage pairs available under four-probe scheme (ordinate). To provide more clarity of the optimal set, the electrode pairs are grouped by their position: $S_{1}$-same side on top surface, $S_{2}$-same side on bottom face, $S_{3}$-opposite faces with oblique left-to-right, and $S_{4}$-opposite faces with oblique right-to-left pairs. All possible combinations have a color on the heatmap. As in the previous case, the EI values of eliminated electrode pairs are set to zero (shown in dark blue).

For the four-probe method, the voltage sites are restricted to lie between the current injection sites. Furthermore, the current injection site itself is not used as a voltage site. This reduces the number of possible combinations. The possible combinations are marked by color on the plots (Figures 6 and 7).

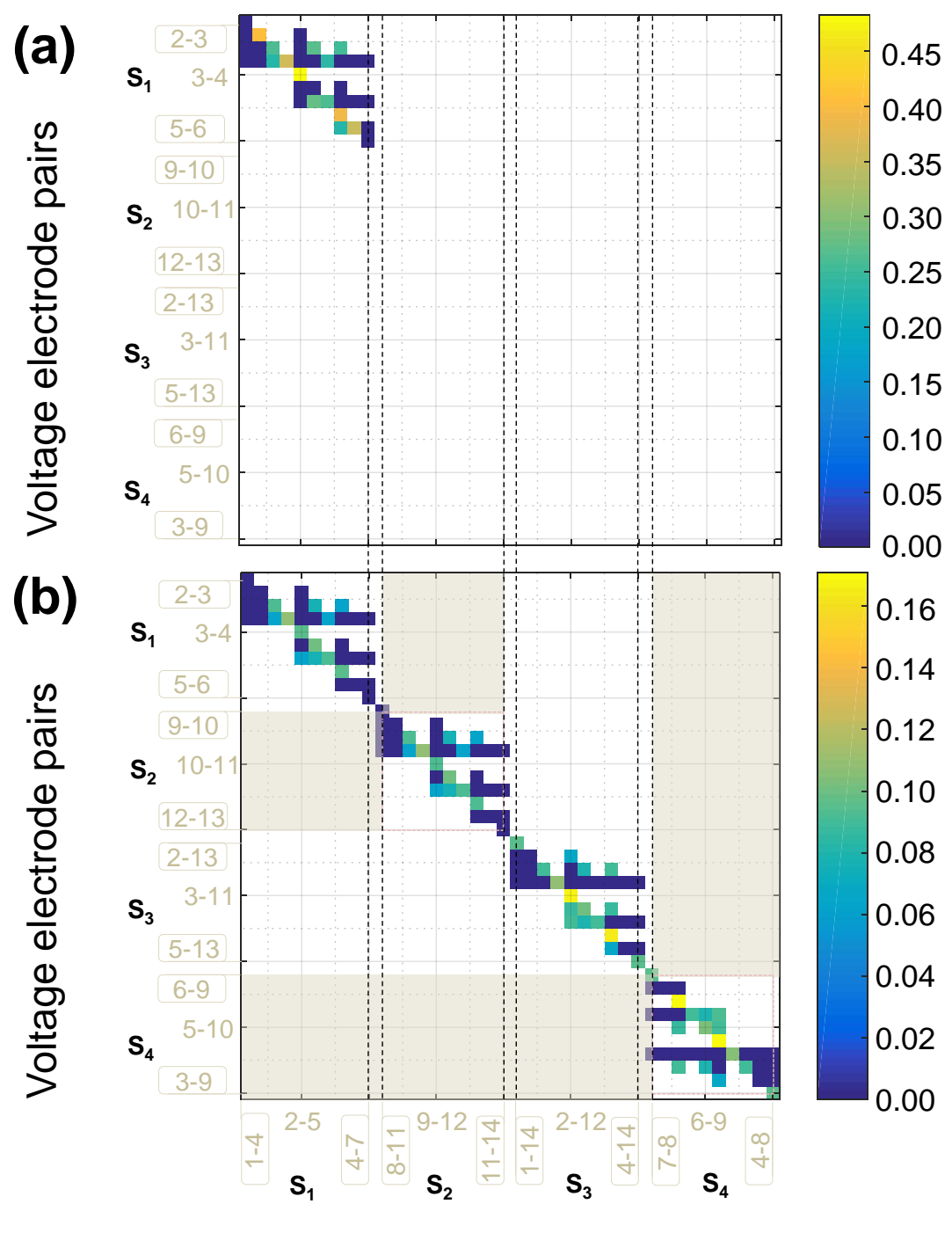

\section{Electric current electrode pairs}

Figure 6. Results of Effective Independence on four-probe schemes on $\left[(0 / 90)_{4}\right]_{s}$ layup using (a) one-sided electrodes and (b) two-sided electrodes. Column sections show groups for surface resistance $\left(S_{1}, S_{2}\right)$ and oblique resistance $\left(S_{3}, S_{4}\right)$ measurements. Cells in dark blue indicate eliminated combination with low EI value.

Using four-probe schemes on the $\left[(0 / 90)_{4}\right]_{s}$ laminate results in a reduced set with 13 out of 35 electrode combinations for one-sided electrodes (Figure 6a) and a reduced set with 55 out of 140 combinations for two-sided electrodes (Figure 6b). For the one-sided setting, the retained optimal 
electrode pairs correspond to pairs with the largest separation. However, the highest contribution is found for the electrode pairs with the lowest separations between current injection electrode pairs and voltage pairs. For the two-sided setting, the preserved combinations are associated with evenly distributed groups of surface and oblique resistance with the highest contributions in the latter (e.g., sections $S_{3}$ and $S_{4}$ in Figure $6 \mathrm{~b}$ ).

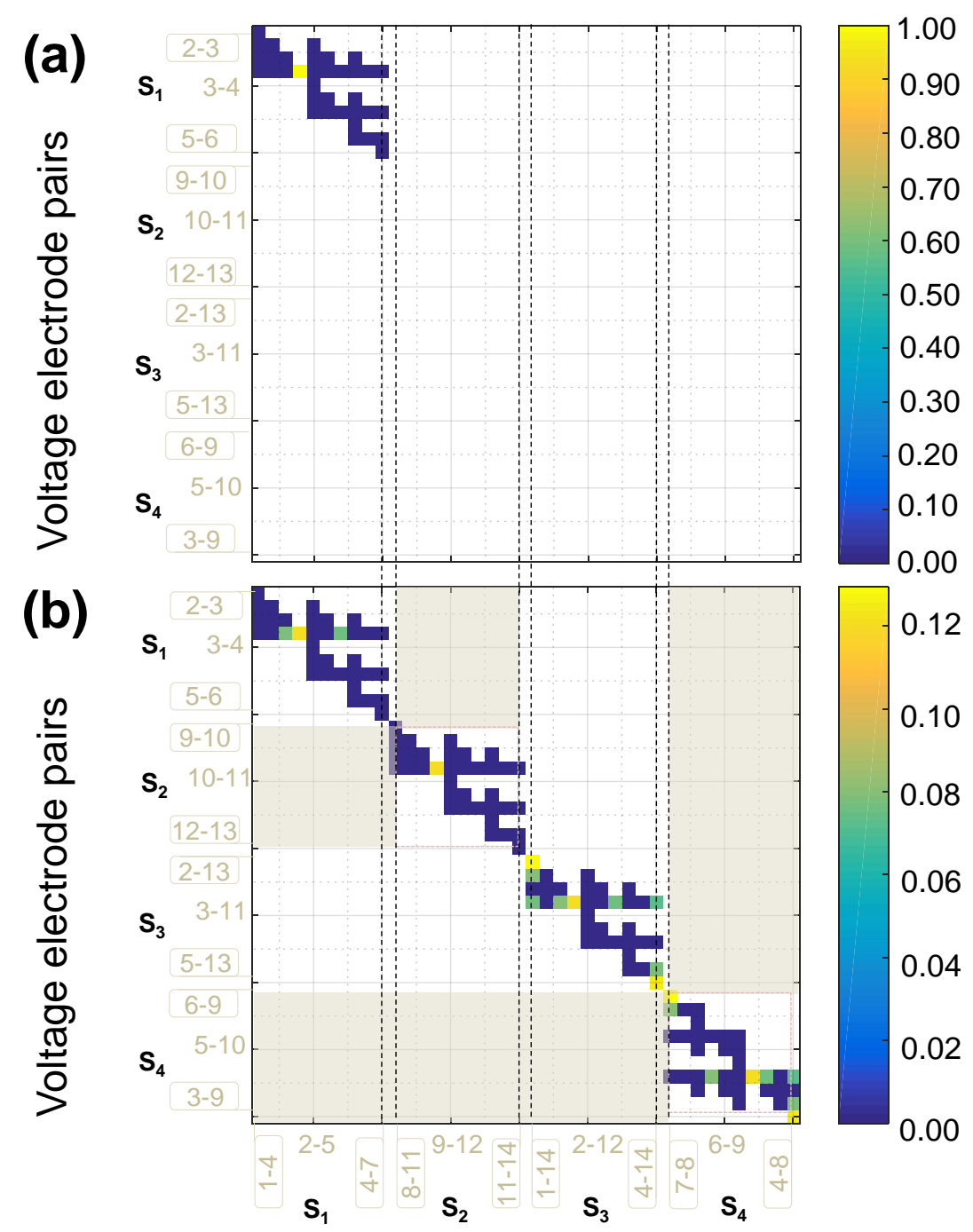

\section{Electric current electrode pairs}

Figure 7. Results of Effective Independence on four-probe schemes on $\left[0_{4} / 90_{4}\right]_{s}$ layup using (a) one-sided electrodes and (b) two-sided electrodes. Column sections show groups for surface resistance $\left(S_{1}, S_{2}\right)$ and oblique resistance $\left(S_{3}, S_{4}\right)$ measurements. Cells in dark blue indicate eliminated combination with low EI value.

For the $\left[0_{4} / 90_{4}\right]_{s}$ laminate under four-probe schemes, when using one-sided electrodes, only one combination is retained. This corresponds to the electrode pairs with the highest separation for current injection and voltage measurement. When using two-sided electrodes, 21 of 140 combinations are retained. The retained electrode pairs with highest contributions correspond to the oblique pairs $S_{3}$ and $S_{4}$ (Figure $7 \mathrm{~b}$ ).

Figures 8 and 9 show the EI values for the reduced and optimum set of responses using a multi-probe scheme. The results are summarized in a $91 \times 14$ grid heatmap plot, with the current injection sites along the abcissa and the voltage electrodes along the ordinate axis. The retained 
combinations for one-sided electrodes are shown in Figures 8a and 9a and the retained combinations for two-sided electrodes are shown in Figures $8 \mathrm{~b}$ and $9 \mathrm{~b}$. In the EI heatmap plots, the electrode pairs used for current injection are arranged into four major groups: $G_{1}$ with electrode pairs on the top surface, $G_{2}$ with electrode pairs on the bottom surface, $G_{3}$ with electrode pairs facing perpendicularly across the thickness and $G_{4}$ with electrode pairs in opposite surfaces facing obliquely. Within each group, the electrode pairs are arranged by the horizontal distance between them in ascending order. This means that, in the $G_{1}$ category, the electrode pairs next to each other $(1-2,2-3,3-4,4-5,5-6,6-7)$ go first followed by $(1-3,2-4,3-5,4-6,5-7)$ and so on until the last 1-7 pair. This is also followed for the $G_{2}$ group. In the $G_{4}$ oblique group, the first half are electrode pairs with top left to bottom right orientation arranged in increasing order of horizontal distance between electrodes, and the second half is the top right to bottom left orientation pairs also arranged in increasing order of distance between the electrodes.

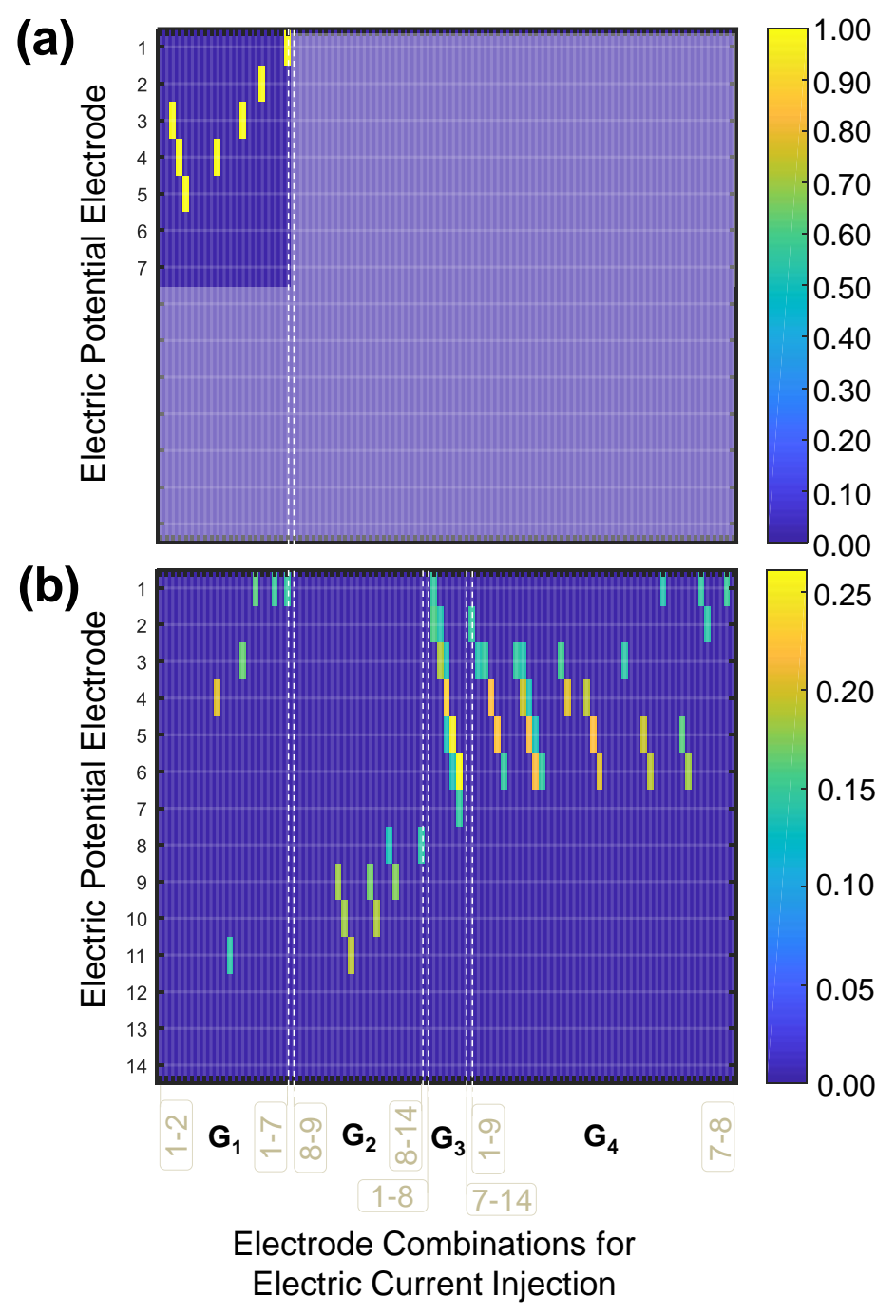

Figure 8. Results of Effective Independence on multi-probe schemes $\left[(0 / 90)_{4}\right]_{S}$ layup using (a) one-sided electrodes and (b) two-sided electrodes.

Electrode combination reduction on the multi-probe schemes using one-sided electrodes retains seven out of 126 combinations for the $\left[(0 / 90)_{4}\right]_{s}$ laminate, and six out of 126 combinations for the $\left[0_{4} / 90_{4}\right] \mathrm{s}$ laminate are retained using one-sided electrodes. For both layups, the retained combinations were those in which electric potential is measured at the electrode where electric current is injected, which is equivalent to a direct resistance measurement (as in a two-probe scheme). 
Electrode combination reduction in multi-probe schemes retains 53 out of 1183 combinations for the $\left[(0 / 90)_{4}\right]_{s}$ laminate and 14 out 1183 combinations for the $\left[0_{4} / 90_{4}\right] s$ laminate, with two-sided electrodes. In both layups, most of the retained combinations were also those related to direct resistance measurement. These oblique resistance measurements were found to have the largest contributions in the multiprobe scheme with two-sided electrodes.

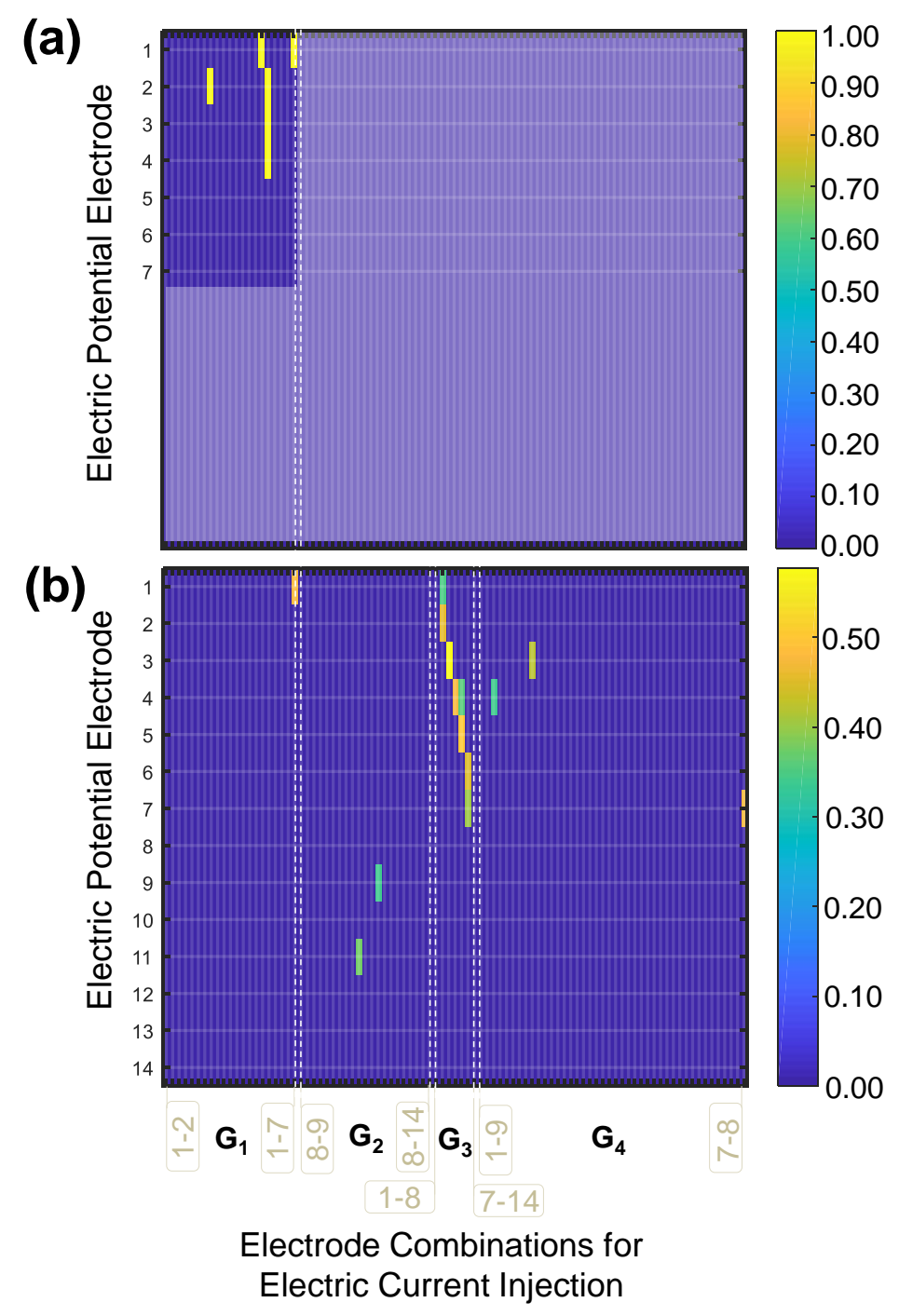

Figure 9. Results of Effective Independence on multi-probe schemes on $\left[0_{4} / 90_{4}\right]_{s}$ layup using (a) one-sided electrodes and (b) two-sided electrodes.

\subsection{Inverse Identification Accuracy with Electrode Selection}

To assess the effectiveness of the EI based sensor selection, we perform inverse identification with the full set and the EI based optimal selected set of electrodes. The inverse identification procedure used is based on a surrogate based optimization approach previously demonstrated successfully for ERT problems by the authors [11,32]. In this procedure, we minimize computational effort by fitting a Kriging surrogate model to the voltage responses obtained by a FEA model. The responses obtained for damage cases created using a full factorial design of experiments ( 810 cases) by varying crack size, and crack $x$ - and $y$-locations were used in the Kriging model fit. This eliminates the use of a FEA model in the inverse identification optimization directly. A separate set of 40 test cases derived from a Latin Hypercube Sampling (LHS) DOE of the damage variable space was analyzed using FEA to obtain the 
needed voltage/resistance responses. These responses are used in lieu of test data as synthetic test data to see how well the inverse optimization can identify these damage cases. The optimization minimizes the $L_{2}-$ norm of the difference between the synthetic responses (simulated measurements) and the predicted responses (from the surrogate). Note that the surrogate Kriging model can introduce a small modeling error, so model and synthetic data can differ even at the same points. The RMS value of the leave one out cross validation errors for the Kriging model were of the order of $5 \%$ or less [32]. The max local errors were of the order of $10 \%$. The inverse optimization does not use any regularization as it only has a small number of variables (the crack parameters), unlike ERT where the description of the entire conductivity field is used as the design variables. Global optimization with Genetic Algorithms (GA) is used.

The accuracy of the inverse identification on 40 test cases for the full set of electrode measurements and reduced set for all three measuring schemes is summarized in Table 2. Each test case uses three damage descriptors, namely: size, $x$-location and $y$-location. The inverse identification predicts the value at each descriptor for every test case. The relative error (RE) between the actual $\left(x_{j}\right)$ and the predicted $\left(\hat{x}_{j}\right)$ value at each damage descriptor is computed and the maximum relative error obtained is used to assess the inverse identification accuracy for a given test case according to Equation (10):

$$
R E(\%)=\operatorname{Max}_{j}\left|\left(\frac{x_{j}-\hat{x}_{j}}{x_{j}^{\text {ref }}}\right)\right| \times 100 \% .
$$

The RE for crack size are calculated relative to the electrode center to center spacing equal $\left(x_{1}{ }^{r e f}=16.5 \mathrm{~mm}\right)$. The RE for the horizontal spacing are calculated relative to the range of $x_{2}$ used for the DOE points $\left(x_{2}{ }^{r e f}=x_{2}{ }^{U}-x_{2}{ }^{L}=69-19.5=49.5 \mathrm{~mm}\right)$. The RE for vertical location are computed relative to the total number of plies $\left(x_{3}{ }^{r e f}=16\right)$. The Root Mean Square (RMS) of the maximum relative errors between the damage descriptors and their predicted values found by inverse identification for the 40 cases is computed.

Table 2. Comparison of inverse identification accuracy for different electrode schemes (for $\left[(0 / 90)_{4}\right]_{S}$ and $\left[0_{4} / 90_{4}\right]_{s}$ layups with full and reduced sets of measurements).

\begin{tabular}{|c|c|c|c|c|c|c|}
\hline \multirow[b]{2}{*}{$\begin{array}{l}\text { Scheme, } \\
\text { Setting }\end{array}$} & \multicolumn{3}{|c|}{$\left[(0 / 90)_{4}\right]_{s}$ Layup } & \multicolumn{3}{|c|}{$\left[0_{4} / 90_{4}\right]_{s}$ Layup } \\
\hline & $\begin{array}{l}\text { No. of } \\
\text { Measurements } \\
\text { Used }\end{array}$ & $\begin{array}{l}\text { No. of Failed } \\
\text { Cases (out of } 40 \\
\text { Test Cases) }\end{array}$ & $\begin{array}{c}\text { RMS of } \\
\text { Inv. Id. } \\
\text { Errors (\%) }\end{array}$ & $\begin{array}{c}\text { No. of } \\
\text { Measurements } \\
\text { Used }\end{array}$ & $\begin{array}{l}\text { No. of Failed } \\
\text { Cases (out of } 40 \\
\text { Test Cases) }\end{array}$ & $\begin{array}{c}\text { RMS of } \\
\text { Inv. Id. } \\
\text { Errors (\%) }\end{array}$ \\
\hline $2 \mathrm{P}, 07 \mathrm{EF}$ & 21 & 12 & 18.29 & 21 & 10 & 14.61 \\
\hline $2 \mathrm{P}, 07 \mathrm{ER}$ & 12 & 11 & 13.77 & 13 & 15 & 20.69 \\
\hline $2 \mathrm{P}, 14 \mathrm{EF}$ & 91 & 1 & 6.75 & 91 & 5 & 6.77 \\
\hline 2P, 14ER & 34 & 1 & 5.17 & 32 & 5 & 7.83 \\
\hline $4 \mathrm{P}, 07 \mathrm{EF}$ & 35 & 14 & 19.60 & 35 & 13 & 25.83 \\
\hline 4P, 07ER & 13 & 17 & 22.54 & 1 & 39 & 60.19 \\
\hline $4 \mathrm{P}, 14 \mathrm{EF}$ & 140 & 2 & 5.05 & 140 & 0 & 2.64 \\
\hline $4 \mathrm{P}, 14 \mathrm{ER}$ & 55 & 3 & 5.18 & 21 & 2 & 4.18 \\
\hline MP, 07EF & 126 & 10 & 16.45 & 126 & 16 & 22.53 \\
\hline MP, 07ER & 7 & 12 & 16.52 & 6 & 16 & 28.37 \\
\hline $\mathrm{MP}, 14 \mathrm{EF}$ & 1,183 & 3 & 5.23 & 1,183 & 4 & 6.50 \\
\hline MP, 14ER & 53 & 1 & 5.09 & 14 & 5 & 6.71 \\
\hline $\begin{array}{l}\text { Scheme 2P: } \\
\text { Scheme 4P: } \\
\text { Scheme MP }\end{array}$ & $\begin{array}{l}\text {-probe measureme } \\
\text {-probe measurem } \\
\text { lti-probe measure }\end{array}$ & & & & & \\
\hline $\begin{array}{l}\text { Setting 07EF } \\
\text { Setting 07EI } \\
\text { Setting 14EF } \\
\text { Setting 14EF }\end{array}$ & $\begin{array}{l}\text { ctrodes on one sic } \\
\text { ctrodes on one si } \\
\text { ctrodes on both si } \\
\text { ctrodes on both si }\end{array}$ & $\begin{array}{l}\text { ull set of electrode } \\
\text { educed set of elec } \\
\text { full set of electroc } \\
\text { reduced set of el }\end{array}$ & $\begin{array}{l}\text { ir combinati } \\
\text { de pair comb } \\
\text { pair combina } \\
\text { code pair con }\end{array}$ & $\begin{array}{l}\text { tions } \\
\text { ns } \\
\text { nations }\end{array}$ & & \\
\hline
\end{tabular}

In addition, the number of cases with relative errors in inverse identification that were larger than $10 \%$ are considered as failed cases. The total number of failed cases and the computed RMS values at each set using the specified scheme and setting shown in Table 2 are used for comparison. 


\subsubsection{Two-Probe Schemes}

The results for the $\left[(0 / 90)_{4}\right]_{s}$ layup using the two-probe scheme with one-sided electrodes show that, for the full set of measurements, out of the 12 failed cases, $100 \%$ are associated with error in crack size identification. However, crack $y$-location identification also fails for $83.33 \%$ of these cases with an average error magnitude of approximately $13 \%$ equivalent to the resolution for 2 -ply. For the reduced set of measurements, $90.1 \%$ of the 11 failed cases are associated with error in crack size identification, whereas the remaining $9.9 \%$ are associated with error in crack $y$-location identification. However, error in the crack location identification is also larger than $10 \%$ in $72.7 \%$ of the failed cases with an average error magnitude of $15.6 \%$ equivalent to the resolution of 2-ply.

The two-probe scheme on the $\left[(0 / 90)_{4}\right]_{s}$ layup with two-sided electrodes and the full set of measurements results in one failed case associated with error in crack size identification. Besides error in crack size, the largest magnitudes are also associated with error in $y$-location identification equivalent to the resolution of 1-ply. For the reduced set of measurements, the only case that fails is associated with error in crack size. The large magnitudes in crack size identification errors are followed by the errors in $y$-location identification, equivalent to the resolution of 1-ply. The failed case is the same for both sets (see Figure 10a,b).
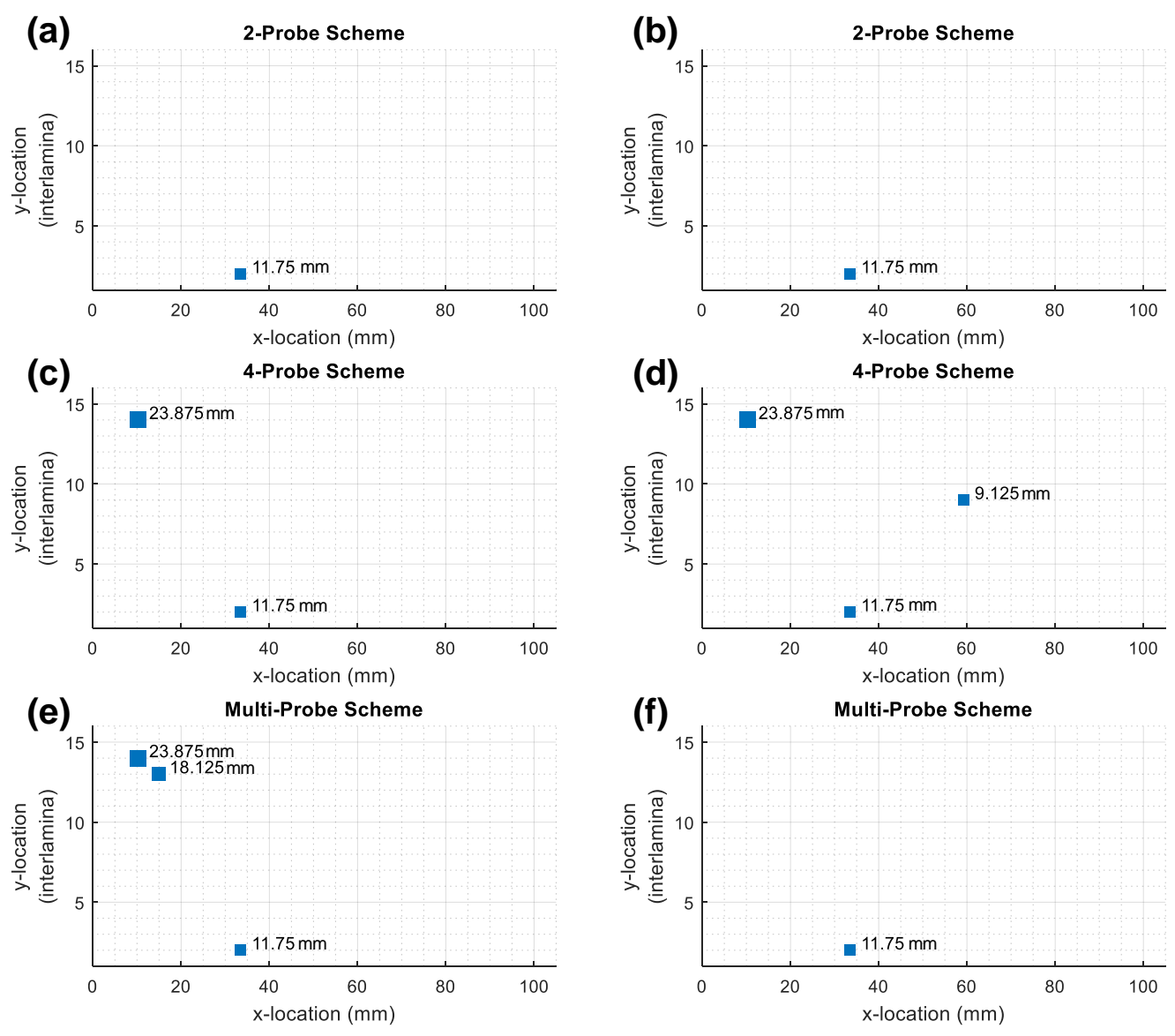

Figure 10. Failed damage cases for $\left[(0 / 90)_{4}\right]_{s}$ with two-sided electrodes and: (a) two-probe scheme and full set of responses; (b) two-probe scheme and reduced set of responses; (c) four-probe scheme and full set of responses; (d) four-probe scheme and reduced set of responses; (e) multi-probe scheme and full set of responses; (f) multi-probe scheme and reduced set of responses. Crack size is shown next to the case box.

The results for the $\left[0_{4} / 90_{4}\right]_{s}$ layup using the two-probe and one-sided electrodes show that, for the full set, 10 cases failed and $80 \%$ of them were associated with error in crack size and $20 \%$ with error 
in $y$-location identification. However, crack $y$-location identification also fails in $70 \%$ of these cases with an average error magnitude of $16 \%$ equivalent to approximately the resolution for 2-ply. For the reduced set, $75 \%$ of the 15 failed cases are associated with error in $y$-location identification and $25 \%$ with crack size identification. However, crack size identification shows errors larger than $10 \%$ in $37.5 \%$ of the failed cases with an average error magnitude of $27 \%$.

Using two-sided electrodes in a two-probe scheme on the $\left[0_{4} / 90_{4}\right]_{s}$ laminate has a total of five failed cases out of the total 40 test cases. Of these five cases, two failed cases (40\%) correspond to crack size identification and three $(60 \%)$ failed in $y$-location identification. The average error in crack size identification was $13 \%$ and the $y$-location error equivalent to 3-ply. For the reduced set of measurements, a total of five cases failed. Two out of the five failed cases $(40 \%)$ are associated with errors in $y$-location identification and three out of five failed cases $(60 \%)$ were for errors in crack size identification. The errors in crack size identification for failed cases were on the order of $17 \%$, while the $y$-location errors were on the order of 3-ply thicknesses. The failed cases with one exception were the same for both sets (see Figure 11a,b).
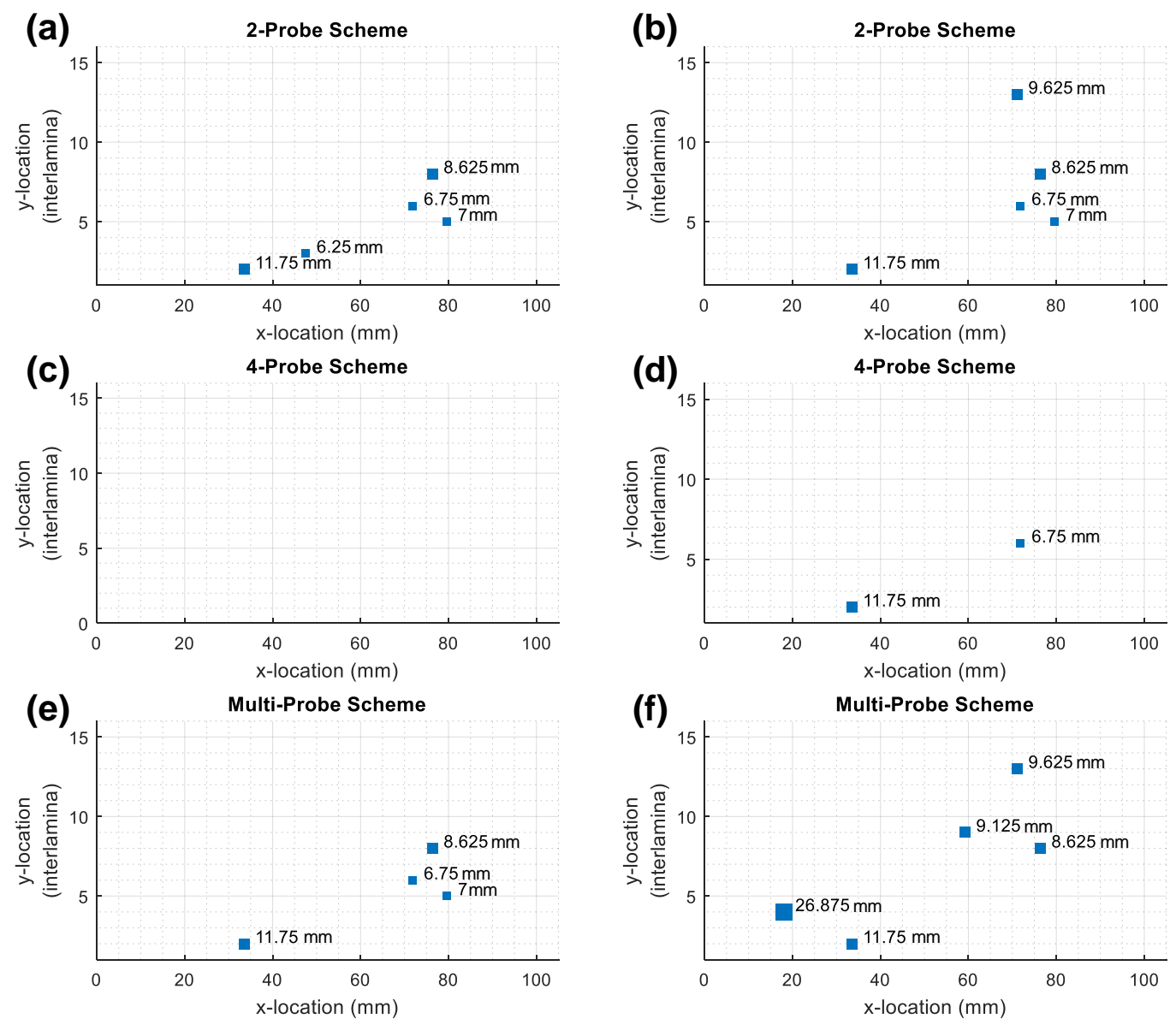

Figure 11. Failed damage cases for $\left[0_{4} / 90_{4}\right]_{S}$ with two-sided electrodes and: (a) two-probe scheme and full set of responses; (b) two-probe scheme and reduced set of responses; (c) four-probe scheme and full set of responses; (d) four-probe scheme and reduced set of responses; (e) multi-probe scheme and full set of responses; (f) multi-probe scheme and reduced set of responses. Crack size is shown next to the case box.

\subsubsection{Four-Probe Schemes}

The results of using the four-probe scheme on the $\left[(0 / 90)_{4}\right]_{S}$ layup and one-sided electrodes result in 14 failed cases out of the total 40 test cases for the inverse optimization using the full set of 
measurements. In these cases, $78.57 \%$ (11 out of 14 ) are associated with identification error in crack size and $21.43 \%$ (three out of 14 ) are associated with errors in $y$-location identification. Error in crack $y$-location identification exceeds $10 \%$ in $64.29 \%$ (nine out of 14 ) of the failed cases and an average error of $20.14 \%$, approximately equivalent to 3-ply. For the reduced set of measurements, inverse identification failed for 17 cases out of the 40 test cases. Of these 17 cases, 14 cases or $82.35 \%$ are associated with large errors in crack size identification and three out of $17(17.65 \%)$ with $y$-location. For the failed cases, crack $y$-location identification error exceeds $10 \%$ in $76.47 \%$ (13 out of 17) of the cases with average error magnitude of $23.08 \%$, which is equivalent to approximately 4 -ply.

The four-probe scheme applied on the $\left[(0 / 90)_{4}\right]_{s}$ layup using two-sided electrodes for the full set of measurements results in only two failed cases among the 40 test cases, both associated with large errors in crack size identification. Errors in $y$-location identification were on the order of 1-ply. In the inverse identification with a reduced set of measurements, only three cases out of the total 40 test cases failed. Two failed cases are associated with error in crack size identification and one with error in $y$-location identification. The errors associated with $y$-location are on the order of 1-ply thickness. The failed cases with one exception are the same for both sets (see Figure $10 \mathrm{c}, \mathrm{d}$ ). One of the failed cases coincides with the failed case from the two-probe scheme.

Inverse identification using the four-probe scheme on the $\left[0_{4} / 90_{4}\right]_{s}$ layup and one-sided electrodes with the full set of measurements results in 13 failed cases among the total 40 test cases. In the failed cases, $53.85 \%$ (seven out of 13 ) are associated with crack size identification errors and $46.15 \%$ (six out of 13) are associated with $y$-location errors. Inverse identification error in crack $y$-location exceeds $10 \%$ in $76.92 \%$ (10 out of 13) of the failed cases with an average error of $18.75 \%$ equivalent to approximately 3 -ply. For the reduced set of measurements, almost all cases fail (39) with $58.97 \%$ of them associated with error in crack size and the rest with errors in $x$-location and $y$-location.

The four-probe scheme applied on the $\left[0_{4} / 90_{4}\right]_{s}$ layup using two-sided electrodes for the full set of measurements results in no failed cases. Some cases have prediction errors larger than $5 \%$ associated with error in crack size and $y$-location identification. For the reduced set of measurements, only two cases failed, one is associated with crack size and one with $y$-location identification. The failed cases in the reduced set (see Figure 11d) coincide with two of the failed cases from the two-probe scheme.

\subsubsection{Multi-Probe Schemes}

Using multi-probe schemes on the $\left[(0 / 90)_{4}\right]_{s}$ layup with one-sided electrodes for the full set of measurements results in 10 failed cases, of which $90 \%$ (nine out of 10) are associated with error in crack size identification and 10\% (one out of 10 failed cases) with $y$-location identification. Among the failed cases, crack $y$-location identification error exceeds $10 \%$ in $80 \%$ of them with average error magnitude of $18 \%$ equivalent to approximately 3-ply. Inverse identification using the reduced set resulted in 12 failed cases, of which $83.33 \%$ are associated with errors in crack size identification and $16.67 \%$ with errors in $y$-location identification. The crack $y$-location identification error exceeds $10 \%$ in $75 \%$ of the failed cases with an average error magnitude of $17.36 \%$ equivalent to approximately 3-ply.

Inverse identification with two-sided electrodes on a $\left[(0 / 90)_{4}\right]_{s}$ laminate with multi-probe schemes using the full set of measurements results in three failed cases. These cases are associated with crack size identification error. Besides crack size, the largest errors found in $y$-location identification were equivalent to the resolution of 1-ply. For the reduced set, only one case fails and it is associated with error in crack size identification. The largest errors in $y$-location identification are equivalent to the resolution of 1-ply. The failed case in the reduced set also fails for the full set (see Figure 10e,f). This case corresponds to delamination near the top surface under one electrode and fails for all sets and schemes in this layup.

Using multi-probe schemes on the $\left[0_{4} / 90_{4}\right]_{s}$ layup with one-sided electrodes for the full set of measurements results in 16 failed cases, of which $56.25 \%$ (nine out of 16) are associated with error in $y$-location identification and $43.75 \%$ (seven out of 16 ) with crack size identification. Inverse identification with the reduced set of measurements results in 16 failed cases, $68.75 \%$ (11 out of 16) 
are associated with error in $y$-location identification and 31.25\% (five out of 16) with error in crack size identification.

Using multi-probe schemes with two-sided electrodes for inverse identification on the $\left[(0 / 90)_{4}\right]_{s}$ laminate with the full set of electrodes results in four failed cases out of 40 test cases-of which, two are associated with crack size identification and two with $y$-location identification. The largest errors found for $y$-location identification are equivalent to the resolution of 1-ply. Inverse identification with the reduced set of measurements results in five failed cases, of which three are associated with $y$-location identification and two with crack size. The largest errors below the $10 \%$ limit are associated with $y$-location identification and are equivalent to the resolution of 1-ply. Two failed cases are found to be common between the complete and reduced set (see Figure 11e,f). These two cases correspond to damage near the top surface under one electrode and delamination in the horizontal midplane. The failed case near the top surface is common to all the schemes and layups, whereas the case in the midplane is only found in the two-probe and multi-probe schemes.

\section{Conclusions}

Electrode pair ranking based on EI measures for damage identification using ERT is influenced by the measuring scheme considered. When using the two-probe scheme, surface resistance measurements of consecutive electrode pairs will have the highest importance followed by through-thickness resistance. In four-probe and multi-probe schemes, oblique resistance is the measurement with more importance.

The number of selected sensing locations using Effective Independence reduction is influenced by the layup when applying four-probe schemes with electrodes on one and both surfaces of the laminate and multi-probe schemes with electrodes on both faces. This influence is not observed when using two-probe schemes.

Using electrodes on both surfaces of the laminate provides better damage identification than using electrodes on one face only. This is made evident for the full and reduced sets of electric measurements. Using sensing electrodes on only one face provides poor accuracy for identification of crack size for both layups using the three measuring schemes. Crack horizontal location is the most accurately identified variable with exception of the $\left[0_{4} / 90_{4}\right]_{s}$ using the four-probe scheme. Vertical location identification accuracy depends on the stacking sequence and measuring scheme. The best performance is for the two-probe method applied on the $\left[(0 / 90)_{4}\right]_{s}$ layup, but, in general, crack vertical location identification errors are larger than two laminae thickness.

ERT damage identification applying EI based reduction of measurements provides similar performance in terms of relative errors when compared to using the complete set of measurements. This is confirmed for the analyzed layups using two-probe, four-probe and multi-probe schemes.

Author Contributions: L.W.E.-G., P.D.-M. and S.V worked on the Forward Problem formulation. L.W.E.-G. worked on the forward problem solving using ANSYS. S.V. and L.W.E.-G. worked on the application of the sensor location optimization criterium to the ERT problem. L.W.E.-G. worked on the Effective Independence optimization implementation and automation. P.D.-M. and S.V. worked on the formulation of the surrogate model and inverse identification process used for validation. P.D.-M. worked on the implementation of this validation. L.W.E.-G. wrote all the sections of this article and S.V. collaborated in the editing process.

Acknowledgments: This research was supported by the Computational Science Research Center (CSRC) at San Diego State University (SDSU).

Conflicts of Interest: The authors declare no conflict of interest. 


\section{Abbreviations}

The following abbreviations are used in this manuscript:

$\begin{array}{ll}\text { CFRP } & \text { Carbon Fiber Reinforced Polymer } \\ \text { ERT } & \text { Electrical Resistance Tomography } \\ \text { EIT } & \text { Electrical Impedance Tomography } \\ \text { EI } & \text { Effective Independence } \\ \text { FIM } & \text { Fisher Information Matrix } \\ \text { FEA } & \text { Finite Element Analysis } \\ \text { FF } & \text { Full Factorial } \\ \text { APDL } & \text { ANSYS Parametric Design Language } \\ \text { GA } & \text { Genetic Algorithm } \\ \text { DOE } & \text { Design of Experiments } \\ \text { RE } & \text { Relative Error } \\ \text { LHS } & \text { Latin Hypercube Sampling } \\ \text { RMS } & \text { Root Mean Square } \\ \text { RMSE } & \text { Root Mean Square Error }\end{array}$

\section{References}

1. Baltopoulos, A.; Polydorides, N.; Pambaguian, L.; Vavouliotis, A.; Kostopoulos, V. Damage identification in carbon fiber reinforced polymer plates using electrical resistance tomography mapping. J. Compos. Mater. 2013, 47, 3285-3301. [CrossRef]

2. Todoroki, A.; Tanaka, Y. Delamination identification of cross-ply graphite/epoxy composite beams using electric resistance change method. Compos. Sci. Technol. 2002, 62, 629-639. [CrossRef]

3. Wang, X.; Wang, S. Sensing damage in carbon fiber and its polymer-matrix and carbon-matrix composites by electrical resistance measurement. J. Mater. Sci. 1999, 34, 2703-2713. [CrossRef]

4. Todoroki, A.; Tanaka, Y.; Shimamura, Y. Multi-prove electric potential change method for delamination monitoring of graphite/epoxy composite plates using normalized response surfaces. Compos. Sci. Technol. 2004, 64, 749-758. [CrossRef]

5. Todoroki, M.; Tanaka, Y.; Shimamura, Y. Measurement of orthotropic electric conductance of CFRP laminates and analysis of the effect on delamination monitoring with an electric resistance change method. Compos. Sci. Technol. 2002, 62, 619-628.

6. Todoroki, A.; Tanaka, M.; Shimamura, Y. Electrical resistance change method for monitoring delaminations of \{CFRP\} laminates: effect of spacing between electrodes. Compos. Sci. Technol. 2005, 65, 37-46. [CrossRef]

7. Todoroki, A.; Tanaka, Y.; Shimamura, Y. Delamination monitoring of graphite/epoxy laminated composite plate of electric resistance change method. Compos. Sci. Technol. 2002, 62, 1151-1160. [CrossRef]

8. Wang, S.; Chung, D. Self-sensing of flexural strain and damage in carbon fiber polymer-matrix composite by electrical resistance measurement. Carbon 2006, 44, 2739-2751. [CrossRef]

9. Todoroki, A.; Tanaka, M.; Shimamura, Y. High performance estimations of delamination of graphite/epoxy laminates with electrical resistance change method. Compos. Sci. Technol. 2003, 63, 1911-1920. [CrossRef]

10. Todoroki, A. The effect of number of electrodes and diagnostic tool for monitoring the delamination of $\{$ CFRP\} laminates by changes in electrical resistance. Compos. Sci. Technol. 2001, 61, 1871-1880. [CrossRef]

11. Escalona-Galvis, L.W.; Diaz-Montiel, P.; Venkataraman, S. Optimal Electrode Selection for Electrical Resistance Tomography in Carbon Fiber Reinforced Polymer Composites. Materials 2017, 10, 125. [CrossRef] [PubMed]

12. Todoroki, A.; Omagari, K.; Shimamura, Y.; Kobayashi, H. Matrix crack detection of CFRP using electrical resistance change with integrated surface probes. Compos. Sci. Technol. 2006, 66, 1539-1545. [CrossRef]

13. Nebuya, S.; Noshiro, M.; Yonemoto, A.; Tateno, S.; Brown, B.H.; Smallwood, R.H.; Milnes, P. Study of the optimum level of electrode placement for the evaluation of absolute lung resistivity with the Mk3.5 EIT system. Physiol. Meas. 2006, 27, S129. [CrossRef] [PubMed]

14. Al Hagrey, S. 2D Optimized Electrode Arrays for Borehole Resistivity Tomography and $\mathrm{CO}_{2}$ Sequestration Modelling. Pure Appl. Geophys. 2012, 169, 1283-1292. [CrossRef]

15. Rucker, D. Enhanced resolution for long electrode ERT. Geophys. J. Int. 2012, 191, 101-111. [CrossRef] 
16. Wilkinson, P.; Meldrum, P.I.; Chambers, J.E.; Kuras, O.; Ogilvy, R.D. Improved strategies for the automatic selection of optimized sets of electrical resistivity tomography measurement configurations. Geophys. J. Int. 2006, 167, 1119-1126. [CrossRef]

17. Goes, B.J.M.; Meekes, J.A.C. An Effective Electrode Configuration for the Detection of DNAPLs with Electrical Resistivity Tomography. J. Environ. Eng. Geophys. 2004, 9, 127-141. [CrossRef]

18. Wang, M.; Yin, W.; Holliday, N. A highly adaptive electrical impedance sensing system for flow measurement. Meas. Sci. Technol. 2002, 13, 12. [CrossRef]

19. Wang, B.; Zhang, W.; Huang, Z.; Ji, H.; Li, H. Modeling and optimal design of sensor for capacitively coupled electrical resistance tomography system. Flow Meas. Instrum. 2013, 31, 3-9. [CrossRef]

20. Wang, P.; Guo, B.; Li, N. Multi-index optimization design for electrical resistance tomography sensor. Measurement 2013, 46, 2845-2853. [CrossRef]

21. Cao, Z.; Wang, H.X.; Xu, L. Electrical impedance tomography with an optimized calculable square sensor. Rev. Sci. Instrum. 2008, 79, 10. [CrossRef] [PubMed]

22. Ma, Y.; Wang, H.; Xu, L.A.; Jiang, C. Simulation study of the electrode array used in an ERT system. Chem. Eng. Sci. 1997, 52, 2197-2203. [CrossRef]

23. Sharifi, M.; Yu, W.; Young, B. Towards fault detection of the operation of dairy processing industry tanks using Electrical Resistance Tomography. Food Control 2014, 38, 192-197. [CrossRef]

24. Hyvönen, N.; Seppänen, A.; Staboulis, S. Optimizing Electrode Positions in Electrical Impedance Tomography. SIAM J. Appl. Math. 2014, 74, 1831-1851. [CrossRef]

25. Furman, A.; Ferré, T.P.A.; Warrick, A.W. Optimization of ERT Surveys for Monitoring Transient Hydrological Events Using Perturbation Sensitivity and Genetic Algorithms. Vadose Zone J. 2004, 3, 1230-1239. [CrossRef]

26. Ren, S.; Zhao, J.; Dong, F. Dimensionality reduced simultaneous iterative reconstruction technique for electrical resistance tomography. Flow Meas. Instrum. 2015, 46, 284-291. [CrossRef]

27. Luppi Silva, O.; Gonzalez Lima, R.; Castro Martins, T.; Silva de Moura, F.; Seiji Tavares, R.; Guerra Tsuzuki, M. Influence of current injection pattern and electric potential measurement strategies in electrical impedance tomography. Control Eng. Pract. 2017, 58, 276-286. [CrossRef]

28. Somersalo, E.; Cheney, M.; Isaacson, D. Existence and Uniqueness for Electrode Models for Electric Current Computed Tomography. SIAM J. Appl. Math. 1992, 52, 1023-1040. [CrossRef]

29. $\quad$ ANSYS $^{\circledR}$ Academic Research, Release 15; ANSYS: Canonsburg, PA, USA, 2015.

30. Kammer, D. Sensor placement for on-orbit modal identification and correlation of large space structures. J. Guidance Control Dyn. 1991, 14, 251-259. [CrossRef]

31. Kammer, D.; Tinker, M. Optimal placement of triaxial accelerometers for modal vibration tests. Mech. Syst. Signal Process. 2004, 18, 29-41. [CrossRef]

32. Diaz Montiel, P.; Escalona, L.; Venkataraman, S. Exploration of Surrogate Models for Inverse Identification of Delamination Cracks in Composites using Electrical Resistance Tomography. In Proceedings of the 58th AIAA/ASCE/AHS/ASC Structures, Structural Dynamics, and Materials Conference, AIAA SciTech Forum, Grapevine, TX, USA, 9-13 January 2017.

(C) 2018 by the authors. Licensee MDPI, Basel, Switzerland. This article is an open access article distributed under the terms and conditions of the Creative Commons Attribution (CC BY) license (http:/ / creativecommons.org/licenses/by/4.0/). 